\title{
On the Derivation of the Nonlinear Discrete Equations Numerically Integrating the Euler PDEs*
}

\author{
F.N. KOUMBOULIS ${ }^{a, \dagger}$, M.G. SKARPETIS ${ }^{b}$ and B.G. MERTZIOS ${ }^{c}$ \\ ${ }^{a}$ University of Thessaly, School of Technological Sciences, Department of Mechanical \& Industrial Engineering, Volos, Greece; \\ ${ }^{\mathrm{b}}$ National Technical University of Athens, Department of Electrical and Computer Engineering, Division of Electroscience, Greece; \\ ${ }^{c}$ Democritus University of Thrace, Department of Electrical and Computer Engineering, 67100, Xanthi, Greece
}

(Received 27 September 1997)

\begin{abstract}
The Euler equations, namely a set of nonlinear partial differential equations (PDEs), mathematically describing the dynamics of inviscid fluids are numerically integrated by directly modeling the original continuous-domain physical system by means of a discrete multidimensional passive (MD-passive) dynamic system, using principles of MD nonlinear digital filtering. The resulting integration algorithm is highly robust, thus attenuating the numerical noise during the execution of the steps of the discrete algorithm. The nonlinear discrete equations approximating the inviscid fluid dynamic phenomena are explicitly determined. Furthermore, the WDF circuit realization of the Euler equations is determined. Finally, two alternative MD WDF set of nonlinear equations, integrating the Euler equations are analytically determined.
\end{abstract}

Keywords: Numerical methods, Nonlinear dynamics, Infinite dimensional systems

\section{INTRODUCTION}

The problem of deriving the analytic solution of nonlinear (NL) partial differential equations (PDEs) is a rather difficult task. The problem has not as yet been solved for the majority of cases. For this reason a variety of numerical methods solving discretized versions of the NLPDEs (see e.g. [1-8] and the references therein) have been developed. The solution of such a numerical problem appears to have many applications in fluid mechanics, electromagnetics and thermodynamics. One important set of NLPDEs is that of the Euler equations. The Euler equations describe the dynamics of an inviscid fluid, thus having a variety of applications in fluid mechanics (industrial equipment, flight systems, fan systems, etc.). The numerical solution of this type of equations has attracted considerable attention (see e.g. [1-8]). The first results regarding the numerical integration of the Euler equations

\footnotetext{
* This work has partially been funded by the General Secretariat for Research and Technology, Greece.

${ }^{\dagger}$ Corresponding author. Mail address: 53 Aftokratoros Irakliou St., 15122 Maroussi, Athens, Greece. E-mail: fkms@athena.domi.gr.
} 
via Wave Digital Filters (WDFs) have been reported in $[9,10]$. In [9] the reference circuit is derived and the general guidelines for the derivation of an MD discretization of the Euler equations for a polytropic fluid are presented. In [10], simulation results are presented for the special case of the 3-D (two spatial variables and one time variable) Euler equations.

The discretization of ODEs (Ordinary Differential Equations) via WDFs appears to be a wellestablished technique since the beginning of $70 \mathrm{~s}$ (see [11] and the references therein). The advantage of this technique is mainly based on the preservation of passivity when the continuous system is transformed to the respective discrete approximation. This way the numerical errors in the iteration of the steps of the discrete algorithm, simulating the original system, appear to be attenuated [12]. This distinct advantage of the WDFs has also been transferred to the case of deriving approximate discretizations of PDEs [13-15]. The property of preserving the passivity under discretization appears to be rather important in the case where the equations are NL or more important in the case of NLPDEs. The nonlinearity of the original system accentuates the numerical errors in the iteration of the steps of the discrete algorithm, thus yielding numerical instability. The approximation of NLPDEs via passive WDFs appears to be a difficult problem not always solvable. The present paper is devoted towards this aim.

In particular, the problem of numerically integrating a special case of NLPDEs, namely the Euler equations, is considered. The contribution of the present paper can be summarized into the analytic determination of the nonlinear discrete set of equations approximating the Euler equations for a polytropic fluid and the respective MD WDF equations integrating the Euler equations. The nonlinear discrete set of equations is proved to be passive. Furthermore, the WDF circuit realization of the Euler equations is determined. Finally, two alternative MD WDF set of nonlinear equations, integrating the Euler equations are analytically determined.

\section{THE EULER PARTIAL DIFFERENTIAL EQUATIONS}

Consider an inviscid compressible fluid. The change in the momentum of the fluid flowing through a control volume at any time instant, as well as the dynamics expressing the conservation of mass are governed by the following two partial differential equations [16]:

$$
\begin{gathered}
\frac{\partial q}{\partial \tilde{t}}+q \cdot \nabla q=f-\frac{\nabla p}{\rho}, \\
\frac{\partial \rho}{\partial \tilde{t}}+\nabla \cdot \rho q=0,
\end{gathered}
$$

respectively. The vector $\tilde{t}_{t}=\left[\begin{array}{llll}t_{1} & t_{2} & t_{3} & \tilde{t}\end{array}\right]^{\mathrm{T}}$ is the vector of the three spatial variables $x, y, z$ and the time $\tilde{t}\left(t_{1}=x, t_{2}=y, t_{3}=z\right)$. Based upon this definition the well known nabla operator $\nabla$ is expressed equivalently as follows:

$$
\nabla=\left[\begin{array}{lll}
\frac{\partial}{\partial t_{1}} & \frac{\partial}{\partial t_{2}} & \frac{\partial}{\partial t_{3}}
\end{array}\right]^{\mathrm{T}} .
$$

The velocity vector is denoted by $q=\left[\begin{array}{lll}q_{1} & q_{2} & q_{3}\end{array}\right]^{\mathrm{T}}$, $\rho$ is the density, $f=\left[\begin{array}{lll}f_{1} & f_{2} & f_{3}\end{array}\right]^{\mathrm{T}}$ is the external forces applied to the fluid inside the control volume, $p$ is the pressure of the fluid in the control volume.

In this section three equivalent expressions of the Euler equations (2.1 and 2) will be presented. These equivalent expressions will facilitate the passive discretization algorithm that will be derived. It is mentioned that the steps required for the derivation of the equivalent forms have first been presented in [9]. For presentation purposes the three equivalent expressions will be partitioned into three different subsections.

\subsection{Generalized Time}

It is convenient to start with the transformation of the time variable $\tilde{t}$ to a new variable $t_{4}$ having dimension of a spatial variable [15]. This new variable is defined by means of a function $q_{4}$ equal to the derivative $q_{4}=q_{4}(\tilde{t})=\mathrm{d} t_{4} / \mathrm{d} \tilde{t}$ of the new variable $t_{4}$. The time variable $t_{4}$ can be considered as an arbitrary, for the time being, variable which 
will be specified later in order to facilitate the proof of passivity. Similarly, the function $q_{4}$ is for the time being arbitrary. Hence, the vector $\tilde{t}_{t}$ is transformed to a new vector $t=\left[\begin{array}{llll}t_{1} & t_{2} & t_{3} & t_{4}\end{array}\right]^{\mathrm{T}}$. Using this new vector and the notation

$$
D=\left[\begin{array}{llll}
D_{1} & D_{2} & D_{3} & D_{4}
\end{array}\right]^{\mathrm{T}} ; \quad D_{k}=\frac{\partial}{\partial t_{k}}(k=1,2,3)
$$

Eqs. (2.1) and (2.2) can be rewritten equivalently as follows:

$$
\begin{gathered}
\sum_{k=1}^{4} \rho q_{k} D_{k} q+\nabla p=f \rho, \\
\sum_{k=1}^{3} D_{k}\left(\rho q_{k}\right)+q_{4} D_{4} \rho=0 .
\end{gathered}
$$

\subsection{Normalization}

The goal of this transformation is to introduce new arbitrary parameters in the Euler equations. These parameters will be useful in proving passivity. Consider the identity

$$
\sqrt{x} D(y \sqrt{x})=x D y+\frac{1}{2} y D x
$$

holding true for every real functions $x, y$ with $x$ scalar $(x \geq 0)$ and $y$ vector. The above identity is usually called normalization identity. Define the following normalized quantities

$$
\begin{aligned}
& \hat{q}=\left[\begin{array}{lll}
\hat{q}_{1} & \hat{q}_{2} & \hat{q}_{3}
\end{array}\right]^{\mathrm{T}}=\frac{1}{q_{4} \sqrt{a}} q, \quad \hat{\rho}=\frac{u_{0}^{2}}{p_{0}} \rho, \\
& \hat{p}=\sqrt{a} \frac{p}{p_{0}}, \quad \hat{f}=\left[\begin{array}{lll}
\hat{f}_{1} & \hat{f}_{2} & \hat{f}_{3}
\end{array}\right]^{\mathrm{T}}=\frac{2 \sqrt{a}}{p_{0}} f \rho,
\end{aligned}
$$

where $u_{0}, p_{0}$ are positive constants and $a=a(\tilde{t})$ is an arbitrary function of time. Using (2.5) Eqs. (2.3) and (2.4) take on the form

$$
\begin{gathered}
\sum_{k=1}^{4} \sqrt{\hat{\rho} a \frac{q_{k} q_{4}}{u_{0}^{2}}} D_{k}\left(\hat{q}_{j} \sqrt{\hat{\rho} a \frac{q_{k} q_{4}}{u_{0}^{2}}}\right) \\
+D_{j} \hat{p}=\frac{1}{2} \hat{f}_{j}, \quad j=1,2,3, \\
\sum_{k=1}^{4} D_{k}\left(\hat{\rho} \frac{q_{k}}{q_{4}}\right)=0 .
\end{gathered}
$$

\subsection{Hadamard Transformation}

Due to conservation of energy, physical systems are usually passive. The systems of PDEs describing a physical system does usually not reflect passivity. The property of passivity is necessary for the discretization of the PDEs and the derivation of their numerical solution. This holds true since a passive multidimensional (MD) circuit yield a causal MD wave digital filter (WDF) which is robust and stable [15]. In particular, in order to obtain a MD passive circuit the following Hadamard transformation is applied

$$
t^{\prime}=H^{-1} t,
$$

where $t^{\prime}=\left[\begin{array}{llll}t_{1}^{\prime} & t_{2}^{\prime} & t_{3}^{\prime} & t_{4}^{\prime}\end{array}\right]^{\mathrm{T}}$ and where $H$ is a suitable orthogonal matrix defined by [9]

$$
H=H^{\mathrm{T}}=\frac{1}{2}\left[\begin{array}{rrrr}
1 & -1 & -1 & 1 \\
-1 & 1 & -1 & -1 \\
-1 & -1 & 1 & 1 \\
1 & 1 & 1 & 1
\end{array}\right]
$$

This is a transformation of the coordinate system (including time). Defining the operators

$$
D_{k}^{\prime}=\frac{\partial}{\partial t_{k}^{\prime}} \quad(k=1,2,3,4)
$$

and using the relations (2.9) and (2.10), the following expression is derived

$$
D^{\prime}=H^{\mathrm{T}} D
$$

From (2.11) and (2.9) the operators $D_{i}^{\prime}$ may be expressed in terms, of new derivative operators as follows:

$$
\begin{aligned}
D_{1} & =\frac{1}{2}\left(D_{1}^{\prime \prime}-D_{4}^{\prime \prime}\right), \quad D_{2}=\frac{1}{2}\left(D_{2}^{\prime \prime}+D_{5}^{\prime \prime}\right), \\
D_{3} & =\frac{1}{2}\left(D_{3}^{\prime \prime}-D_{6}^{\prime \prime}\right), \\
D_{4} & =\frac{1}{2}\left(D_{1}^{\prime \prime}+D_{4}^{\prime \prime}\right)=\frac{1}{2}\left(D_{2}^{\prime \prime}+D_{5}^{\prime \prime}\right) \\
& =\frac{1}{2}\left(D_{3}^{\prime \prime}+D_{6}^{\prime \prime}\right)
\end{aligned}
$$


or inversely

$D_{1}^{\prime \prime}=D_{4}+D_{1}, \quad D_{2}^{\prime \prime}=D_{4}+D_{2}, \quad D_{3}^{\prime \prime}=D_{4}+D_{3}$,

$D_{4}^{\prime \prime}=D_{4}-D_{1}, \quad D_{5}^{\prime \prime}=D_{4}-D_{2}, \quad D_{6}^{\prime \prime}=D_{4}-D_{3}$.

The above relations can be considered as a definition of the operators $D_{i}^{\prime \prime}(i=1,2,3,4)$ in terms of the operators $D_{i}(i=1,2,3,4)$. Using the above transformations and after some extensive manipulations Eqs. (2.6) and (2.7) may be rewritten as follows:

$$
\begin{gathered}
\sum_{i=1}^{7} u_{j, i}=\hat{f}_{j}, \quad j=1,2,3 \\
\sum_{i=1}^{10} u_{4, i}=0
\end{gathered}
$$

where the variables $u_{j, i}$ for $j=1,2,3$ are related to the normalized velocities as follows:

$$
\begin{gathered}
u_{j, i}=\sqrt{L_{i}} D_{i}^{\prime}\left(\sqrt{L_{i}} \hat{q}_{j}\right), \quad i=1,2,3,4,5 \\
D_{5}^{\prime}=D_{4} \\
u_{j, 6}=D_{j}^{\prime \prime}\left(\hat{q}_{j}+\hat{p}\right), \quad u_{j, 7}=D_{j+3}^{\prime \prime}\left(\hat{q}_{j}-\hat{p}\right)
\end{gathered}
$$

and for $j=4$, as follows

$$
\begin{gathered}
u_{4, i}=\sqrt{L_{0, i}} D_{i}^{\prime}\left(\sqrt{L_{0, i}} \hat{p}\right), \quad i=1,2,3,4 \\
u_{4, i+4}=u_{i, 6}, \quad u_{4, i+7}=-u_{i, 7}, \quad i=1,2,3
\end{gathered}
$$

The parameters $L_{i}$ and $L_{0, i}$ are given by

$$
\begin{aligned}
L_{i} & =\hat{\rho} a \frac{u_{0, i} q_{4}}{u_{0}^{2}}-\varepsilon, \quad i=1,2,3,4, \\
L_{5} & =2(\varepsilon-1), \\
L_{0, i} & =2 \frac{\nu \hat{\rho}-1}{\hat{p}} \hat{u}_{0, i}-3+\frac{K_{1}}{\hat{p}^{2}}-\varepsilon,
\end{aligned}
$$

where $K_{1}$ and $\varepsilon$ arbitrary real constants with $\varepsilon \geq 1$, and where

$$
\begin{aligned}
& u_{0,1}=q_{1}-q_{2}-q_{3}+q_{4}, \\
& u_{0,2}=-q_{1}+q_{2}-q_{3}+q_{4}, \\
& u_{0,3}=-q_{1}-q_{2}+q_{3}+q_{4},
\end{aligned}
$$

$$
\begin{aligned}
& u_{0,4}=q_{1}+q_{2}+q_{3}+q_{4}, \\
& \hat{u}_{0, i}=\frac{u_{0, i}}{\sqrt{a} q_{4}}, \nu=\frac{1}{p} \int \frac{\mathrm{d} p}{\hat{\rho}}=\frac{p_{0}}{u_{0}^{2} p} \int \frac{\mathrm{d} p}{\rho},
\end{aligned}
$$

where for the derivation of the above relations it has been assumed that the fluid is barotropic, i.e. that the pressure is a unique function of the density. Clearly this assumption covers the majority of fluid mechanics phenomena. The variables $u_{i, j}$, being a nonlinear transformation of the fluid velocities are the unknowns in the linear system of equations (2.15).

\section{GENERALIZED KIRCHHOFF CIRCUIT REALIZATION}

It can easily be observed that the transformed form of the Euler equations, namely the four equations in (2.15) describe a generalized Kirchhoff circuit [9] involving four loops, appropriately interconnected.

The generalized Kirchhoff circuit involving the four loops is shown in Fig. 1. The symbols $L_{i}, D_{i}^{\prime}$ $(i=1,2,3,4,5)$ denote the operator applied to the current $\hat{q}_{j}(j=1,2,3)$ to yield the voltage $u_{j, i}=$ $\sqrt{L_{i}} D_{i}^{\prime}\left(\sqrt{L_{i}} \hat{q}_{j}\right)$ (see also (2.16a)). An analogous definition holds for $L_{0 i}, D_{i}^{\prime}(i=1,2,3,4$ and 5), $D_{i}^{\prime \prime}(i=1,2,3,4,5)$. For more details see relations (2.16c) and (2.16d). The circuit in Fig. 1 is passive if every element of the circuit is positive i.e. if

$$
L_{1}, \ldots, L_{5} \geq 0, \quad L_{01}, \ldots, L_{04} \geq 0 .
$$

To analyze the above conditions consider the case of a polytropic fluid, i.e. a fluid with pressure depending upon the density by a relation of the form: $p=C \rho^{n}$ (where $C, n$ are positive constants real numbers with $n>1$ ). Using the assumption that the fluid is polytropic and after some algebraic manipulations, the conditions in (3.1) can be expressed in form of denormalized variables as in the following Lemma [9]. 


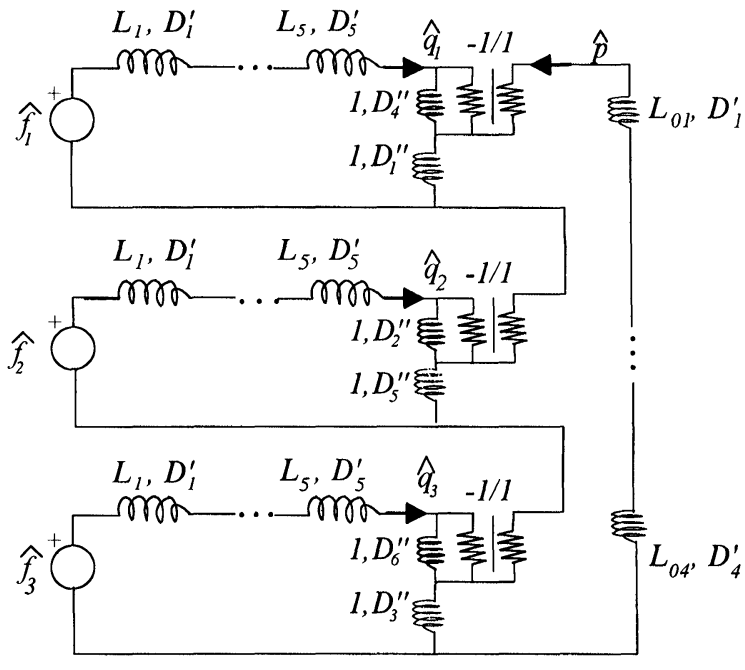

FIGURE 1 Generalized Kirchhoff circuit.

LEMMA 3.1 If the following inequalities are satisfied

$$
\frac{3(n-1) p_{\max }}{2 q_{4}-2 \sqrt{3} q_{\max }} \leq \frac{p_{0}}{a q_{4}} \leq \frac{\rho_{\min }}{\varepsilon}\left(q_{4}-\sqrt{3} q_{\max }\right),
$$

where

$p_{\max }=\max _{t} p(t), q_{\max }=\max _{t} q(t), \rho_{\max }=\max _{t} \rho(t)$

then the circuit of Fig. 1 is passive.

LEMMA 3.2 The inequality in (3.2) is satisfied if the generalized derivative of time $q_{4}$ is chosen to be

$$
q_{4} \geq \sqrt{3} q_{\max }+\sqrt{\frac{3(n-1) p_{\max }}{2 \rho_{\min }}}
$$

while the arbitrary parameters $a$ and $p_{0}$ can be assigned to be

$$
\frac{p_{0}}{a}=q_{4} \sqrt{\frac{3(n-1)}{2 \varepsilon} \rho_{\min } p_{\max }} .
$$

Proof To prove the above relations, substitute (3.3) and (3.4) into (3.2), to yield inequalities identically true.
Combining Lemma 3.1 and Lemma 3.2, the following theorem is established.

THEOREM 3.1 There always exists a passive generalized Kirchhoff circuit realization of the Euler equations.

\section{DISCRETIZATION ALGORITHM}

In order to transform the circuit of Fig. 1 to an equivalent reference WDF circuit the circuit must first be discretized. To this end the trapezoidal rule must be applied (see e.g. [16]) to the inductances occurring in the circuit. With regard to the trapezoidal rule it is important to point out that it is one of the most accurate discretization rules. Consider the case of an one dimension inductance

$$
u=D(L i), \quad D=\frac{\mathrm{d}}{\mathrm{d} \tilde{t}},
$$

where $\tilde{t}$ is the original time variable, $u$ is the voltage, $i$ is the current while $L$ (the inductance coefficient) is a constant. According to the trapezoidal rule, the discretization takes on the form

$$
u(\tilde{t})+u\left(\tilde{t}-\tilde{T}_{0}\right)=\frac{2 L}{\tilde{T}_{0}}\left[i(\tilde{t})-i\left(\tilde{t}-\tilde{T}_{0}\right)\right],
$$

where $\tilde{T}_{0} \in \mathbb{R}$ is the sampling period. The operator mapping the discrete $i$ to the discrete $u$ is denoted by $R \Delta\left(\tilde{T}_{0}\right)$, i.e.

$$
u(\tilde{t})=R \Delta\left(\tilde{T}_{0}\right) i(\tilde{t}), \quad R=2 L / \tilde{T}_{0} .
$$

Since the inductances appearing in Fig. 1, are strongly nonlinearly dependent upon the four generalized spatial variables $t_{1}^{\prime}, t_{2}^{\prime}, t_{3}^{\prime}, t_{4}^{\prime}$ a more generalized treatment has to be applied. Similarly, to [11] consider the operator $\bar{D}=\partial(\cdot) / \partial t_{1}^{\prime}+$ $\partial(\cdot) / \partial t_{2}^{\prime}+\partial(\cdot) / \partial t_{3}^{\prime}+\partial(\cdot) / \partial t_{4}^{\prime}$ differentiating the argument quantity with respect to all elements of the vector $t^{\prime}=\left[\begin{array}{llll}t_{1}^{\prime} & t_{2}^{\prime} & t_{3}^{\prime} & t_{4}^{\prime}\end{array}\right]^{\mathrm{T}}$. Furthermore, consider the voltage, $\bar{u}\left(t^{\prime}\right)$ defined by the following 
generalized inductance description:

$$
\bar{u}=\sqrt{\bar{L}\left(t^{\prime}\right)} \bar{D}\left(\sqrt{\bar{L}\left(t^{\prime}\right)} \bar{i}\left(t^{\prime}\right)\right),
$$

where $\bar{i}\left(t^{\prime}\right)$ is a generalized current. Apply the general trapezoidal rule to the relation (4.3) to yield

$$
\begin{aligned}
& \left(\frac{\bar{u}}{\sqrt{\bar{L}}}\right)\left(t^{\prime}\right)+\left(\frac{\bar{u}}{\sqrt{\bar{L}}}\right)\left(t^{\prime}-T^{\prime}\right) \\
& \quad=\frac{2}{T_{0}}\left[(\sqrt{\bar{L}} \bar{i})\left(t^{\prime}\right)-(\sqrt{\bar{L}} \bar{i})\left(t^{\prime}-T^{\prime}\right)\right]
\end{aligned}
$$

where $T^{\prime}$ is a vector of shift of the sampling having the form

$$
\begin{aligned}
T^{\prime} & =\left[\begin{array}{llll}
T_{1} & T_{2} & T_{3} & T_{4}
\end{array}\right]^{\mathrm{T}}=\alpha T_{0}, \quad T_{0}>0 ; \\
\alpha & =\left[\begin{array}{llll}
\alpha_{1} & \alpha_{2} & \alpha_{2} & \alpha_{3}
\end{array}\right]^{\mathrm{T}}, \quad \alpha_{i} \geq 0 .
\end{aligned}
$$

The above sampling vector can be interpreted as follows: $T_{0}$ is the basic sampling period (scalar and constant), $T^{\prime}=\left[\begin{array}{llll}T_{1} & T_{2} & T_{3} & T_{4}\end{array}\right]^{\mathrm{T}}$ is the vector of sampling period for each transformed space and time coordinate. The coefficients $\alpha_{i}$ are the weight coefficients for every sampling period respectively. It is clear that as $\alpha_{i} \rightarrow 0$ the discretization becomes perfectly accurate. It is important to note that, in many cases, since all elements of $t^{\prime}=\left[\begin{array}{llll}t_{1}^{\prime} & t_{2}^{\prime} & t_{3}^{\prime} & t_{4}^{\prime}\end{array}\right]^{\mathrm{T}}$ are in spatial dimensions the weighting coefficients can be considered to be equal

$$
\alpha_{i}=\alpha^{\prime}, \quad T_{i}=\alpha^{\prime} T_{0}=T_{0}^{\prime} .
$$

The trapezoidal rule can be expressed in terms of an operator, let $\Delta\left(T^{\prime}\right)\{\bar{r} \cdot\}$, as follows

$$
\bar{u}\left(t^{\prime}\right)=\Delta\left(T^{\prime}\right)\left\{\bar{r}\left(t^{\prime}\right) \bar{i}\left(t^{\prime}\right)\right\}, \quad \bar{r}\left(t^{\prime}\right)=\frac{2 \bar{L}\left(t^{\prime}\right)}{T_{0}^{\prime}} .
$$

In order to discretize the inductances in the circuit of Fig. 1, i.e. the inductances

$$
\begin{aligned}
& u_{j, i}=L_{i} D_{i}^{\prime}\left\{\hat{q}_{j}\right\} \quad(i=1,2,3,4,5) \quad(j=1,2,3), \\
& u_{j, 6}=D_{j}^{\prime \prime}\left\{\hat{q}_{j}+\hat{p}\right\} \quad(j=1,2,3), \\
& u_{j, 7}^{\prime}=D_{j+3}^{\prime \prime}\left\{\hat{q}_{j}-\hat{p}\right\} \quad(j=1,2,3), \\
& u_{4, i}=L_{0 i} D_{i}^{\prime}\{\hat{p}\} \quad(i=1,2,3,4),
\end{aligned}
$$

the general trapezoidal rule is applied in accordance to (4.6) to yield

$$
\begin{gathered}
u_{j, i}\left(t^{\prime}\right)=\Delta\left(T_{i}^{\prime}\right)\left\{r_{i}\left(t^{\prime}\right) \hat{q}_{j}\left(t^{\prime}\right)\right\} \\
r_{i}\left(t^{\prime}\right)=\frac{2 L_{i}\left(t^{\prime}\right)}{T_{0}^{\prime}} \\
(i=1,2,3,4,5) \quad(j=1,2,3), \\
u_{i, 6}\left(t^{\prime}\right)=\frac{2}{T_{0}^{\prime}} \Delta\left(T_{j}^{\prime \prime}\right)\left\{\hat{q}_{j}\left(t^{\prime}\right)+\hat{p}\left(t^{\prime}\right)\right\} \quad(j=1,2,3), \\
u_{i, 7}\left(t^{\prime}\right)=\Delta\left(T_{j+3}^{\prime \prime}\right)\left\{\hat{g}_{j}\left(t^{\prime}\right)-\hat{p}\left(t^{\prime}\right)\right\} \quad(j=1,2,3), \\
u_{4, i}\left(t^{\prime}\right)=\Delta\left(T_{i}^{\prime}\right)\left\{r_{0 i}\left(t^{\prime}\right) \hat{p}\right\} \\
r_{0 i}\left(t^{\prime}\right)=\frac{2 L_{0 i}\left(t^{\prime}\right)}{T_{0}^{\prime}} \quad(i=1,2,3,4),
\end{gathered}
$$

where the above shifting vectors (multidimensional shifts) are defined by the relations

$$
\begin{aligned}
& T_{1}^{\prime}=\left[\begin{array}{llll}
T_{0}^{\prime} & 0 & 0 & 0
\end{array}\right]^{\mathrm{T}}, \quad T_{2}^{\prime}=\left[\begin{array}{llll}
0 & T_{0}^{\prime} & 0 & 0
\end{array}\right]^{\mathrm{T}}, \\
& T_{3}^{\prime}=\left[\begin{array}{llll}
0 & 0 & T_{0}^{\prime} & 0
\end{array}\right]^{\mathrm{T}}, \quad T_{4}^{\prime}=\left[\begin{array}{llll}
0 & 0 & 0 & T_{0}^{\prime}
\end{array}\right]^{\mathrm{T}}, \\
& T_{5}^{\prime}=\frac{1}{2}\left[\begin{array}{llll}
T_{0}^{\prime} & T_{0}^{\prime} & T_{0}^{\prime} & T_{0}^{\prime}
\end{array}\right]^{\mathrm{T}}, \\
& T_{1}^{\prime \prime}=\left[\begin{array}{llll}
T_{0}^{\prime} & 0 & 0 & T_{0}^{\prime}
\end{array}\right]^{\mathrm{T}}, \quad T_{2}^{\prime \prime}=\left[\begin{array}{llll}
0 & T_{0}^{\prime} & 0 & T_{0}^{\prime}
\end{array}\right]^{\mathrm{T}}, \\
& T_{3}^{\prime \prime}=\left[\begin{array}{llll}
0 & 0 & T_{0}^{\prime} & T_{0}^{\prime}
\end{array}\right]^{\mathrm{T}} \text {, } \\
& T_{4}^{\prime \prime}=\left[\begin{array}{llll}
0 & T_{0}^{\prime} & T_{0}^{\prime} & 0
\end{array}\right]^{\mathrm{T}}, \quad T_{5}^{\prime \prime}=\left[\begin{array}{llll}
T_{0}^{\prime} & 0 & T_{0}^{\prime} & 0
\end{array}\right]^{\mathrm{T}}, \\
& T_{6}^{\prime \prime}=\left[\begin{array}{llll}
T_{0}^{\prime} & T_{0}^{\prime} & 0 & 0
\end{array}\right]^{\mathrm{T}} \text {. }
\end{aligned}
$$

Based upon the discretization expressions in (4.7) the discretized circuit, corresponding to the passive circuit of Fig. 1, is derived to be the reference circuit in Fig. 2 that follows. Based upon the reference circuit of Fig. 2, the following set of discrete equations is derived for the numerical integration of the Euler equations.

$$
\begin{aligned}
& \sum_{i=1}^{7} u_{j, i}\left(t^{\prime}\right)=\hat{f}_{i}\left(t^{\prime}\right), \quad j=1,2,3, \\
& \sum_{i=1}^{10} u_{4, i}\left(t^{\prime}\right)=0,
\end{aligned}
$$

where the discrete voltages are defined in (4.7). 


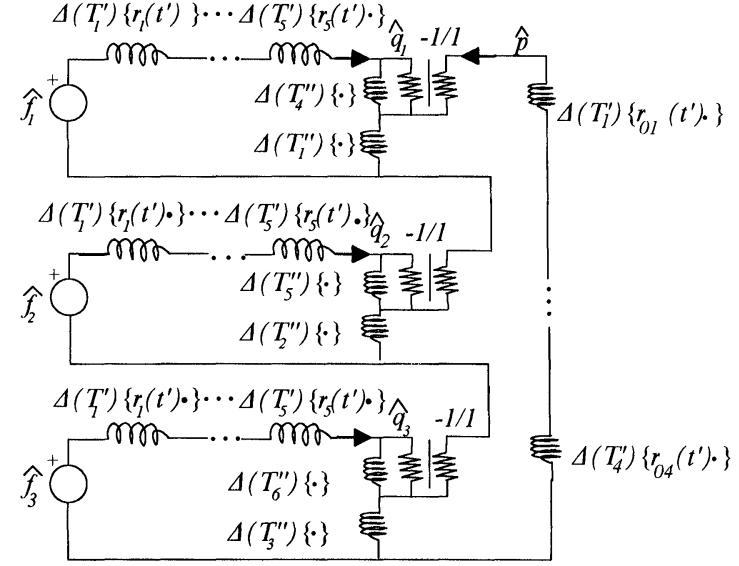

FIGURE 2 Reference circuit.

THEOREM 4.1 The set of nonlinear discrete equations approximating the Euler equations is the multidimensional (MD) system of equations in (2.7) and (2.10).

Remark 4.1 The MD system of equations in (2.7) and (2.10) is in generalized state space solvable form. Thus it can recursively be solved around an area of the generalized time $t^{\prime}$.

\section{WDF REALIZATION}

In this section the analytic and circuit forms of the WDF realization of the reference circuit in Fig. 2, or equivalently of the MD system of equations in (2.7) and (2.10), will be derived. The analytic form of the WDF realization is the formulation of the numerical algorithm integrating the Euler equations. In order to derive a robust algorithm it is suitable to adopt power waves instead of the voltage waves usually preferred in wave digital filtering [11]. Thus for a port of voltage $u$, current $i$ and nonconstant port resistance $R \geq 0$, the forward $a$ and the backward wave $b$ are defined as follows:

$$
a=\frac{u+R i}{2 \sqrt{R}}, \quad b=\frac{u-R i}{2 \sqrt{R}} .
$$

The reference circuit in Fig. 2 can be analyzed into four loops where only the forth loop is connected to the other three. Based upon this observation and

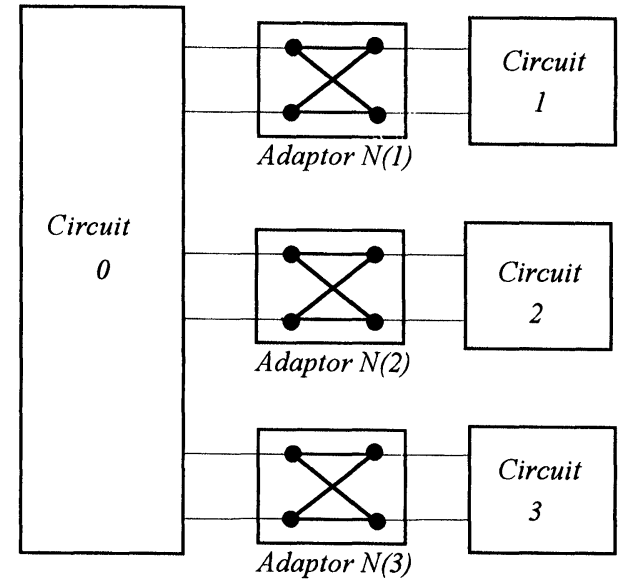

FIGURE 3 WDF arrangement.

the theory of WDF [11], the reference circuit can be realized by four WDF circuits where the fourth is connected to the rest three by appropriate adaptors. Let Circuit $i(i=0,1,2,3)$ be the WDF circuit corresponding to the $i$ th loop of the reference circuit. Let $N(i)(i=1,2,3)$ connecting the Circuit $i$ to the Circuit 0. Hence, the structure of the WDF realization is that presented in Fig. 3. Realizing the Connection $i$ (Adaptor $N(i)$ ) and the Circuit $i$, according to the WDF theory [11], the WDF circuit in Fig. 4 is derived.

The arrangement of Fig. 3 contains 4 series adaptors $(0,1,2,3)$ according to the 4 mail loops in Fig. 2, and additional adaptors marked $N^{\prime}(i)$ and $N^{\prime \prime}(i)(i=1,2,3)$. The required multiplier coefficients in these adaptors can be determined if the corresponding port resistance are determined. According to Fig. 2, and the results presented in [9] the port resistances can be computed to be

$$
\begin{gathered}
R_{i, k}=\frac{2}{T_{0}^{\prime}} L_{k}, \quad R_{i, 6}=\frac{4}{T_{0}^{\prime}}, \\
k=1,2,3,4,5, \quad i=1,2,3 \\
R_{0, k}=\frac{2}{T_{0}^{\prime}} L_{0, k}, \quad R_{0,4+i}=\frac{4}{T_{0}^{\prime}}, \\
i=1,2,3, \quad k=1,2,3,4 \\
R_{i, 0}=\sum_{k=1}^{6} R_{i, k}, \quad i=1,2,3 .
\end{gathered}
$$


According to (4.10), (4.7) and Fig. 4 the forward waves, appearing in the adaptors $(0,1,2,3)$, are expressed in terms of the backward waves at previous "time" instants as follows:

$$
\begin{aligned}
& a_{i, 1}\left(t_{1}^{\prime}, t_{2}^{\prime}, t_{3}^{\prime}, t_{4}^{\prime}\right)=b_{i, 1}\left(t_{1}^{\prime}-T_{0}^{\prime}, t_{2}^{\prime}, t_{3}^{\prime}, t_{4}^{\prime}\right), \\
& \quad i=0,1,2,3, \\
& a_{i, 2}\left(t_{1}^{\prime}, t_{2}^{\prime}, t_{3}^{\prime}, t_{4}^{\prime}\right)=b_{i, 2}\left(t_{1}^{\prime}, t_{2}^{\prime}-T_{0}^{\prime}, t_{3}^{\prime}, t_{4}^{\prime}\right), \\
& \quad i=0,1,2,3, \\
& a_{i, 3}\left(t_{1}^{\prime}, t_{2}^{\prime}, t_{3}^{\prime}, t_{4}^{\prime}\right)=b_{i, 3}\left(t_{1}^{\prime}, t_{2}^{\prime}, t_{3}^{\prime}-T_{0}^{\prime}, t_{4}^{\prime}\right), \\
& \quad i=0,1,2,3, \\
& a_{i, 4}\left(t_{1}^{\prime}, t_{2}^{\prime}, t_{3}^{\prime}, t_{4}^{\prime}\right)=b_{i, 4}\left(t_{1}^{\prime}, t_{2}^{\prime}, t_{3}^{\prime}, t_{4}^{\prime}-T_{0}^{\prime}\right), \\
& \quad i=0,1,2,3, \\
& a_{i, 5}\left(t_{1}^{\prime}, t_{2}^{\prime}, t_{3}^{\prime}, t_{4}^{\prime}\right) \\
& =b_{i, 5}\left(t_{1}^{\prime}-T_{0}^{\prime}, t_{2}^{\prime}-T_{0}^{\prime}, t_{3}^{\prime}-T_{0}^{\prime}, t_{4}^{\prime}-T_{0}^{\prime}\right), \\
& i=1,2,3 .
\end{aligned}
$$

The equations defining the adaptors $N^{\prime}(i)$ and $N^{\prime \prime}(i)(i=1,2,3)$ are

$$
\begin{aligned}
a_{0,4+i}\left(t^{\prime}\right) & =a_{0,4+i}^{\prime}\left(t^{\prime}\right)+a_{i, 6}^{\prime}\left(t^{\prime}\right), \\
a_{i, 6}\left(t^{\prime}\right) & =-a_{0,4+i}^{\prime}\left(t^{\prime}\right)+a_{i, 6}^{\prime}\left(t^{\prime}\right),
\end{aligned}
$$

with

$$
\begin{aligned}
a_{i, 6}^{\prime}\left(t^{\prime}\right) & =b_{i, 6}^{\prime}\left(t^{\prime}-T_{3+i}^{\prime \prime}\right), \\
a_{0,4+i}^{\prime}\left(t^{\prime}\right) & =b_{0,4+i}^{\prime}\left(t^{\prime}-T_{i}^{\prime \prime}\right),
\end{aligned}
$$

where

$$
\begin{aligned}
b_{i, 6}^{\prime}\left(t^{\prime}\right) & =-\frac{1}{2}\left(b_{0,4+i}\left(t^{\prime}\right)+b_{i, 6}\left(t^{\prime}\right)\right), \\
b_{0,4+i}^{\prime}\left(t^{\prime}\right) & =\frac{1}{2}\left(b_{0,4+i}\left(t^{\prime}\right)-b_{i, 6}\left(t^{\prime}\right)\right) .
\end{aligned}
$$

The equations of the three 7-port series adaptors in the Circuits 1, 2 and 3 are

$$
b_{i, k}=a_{i, k}-\gamma_{i, k} \tilde{a}_{i, 0}, \quad i=1,2,3, \quad k=0, \ldots, 6,
$$

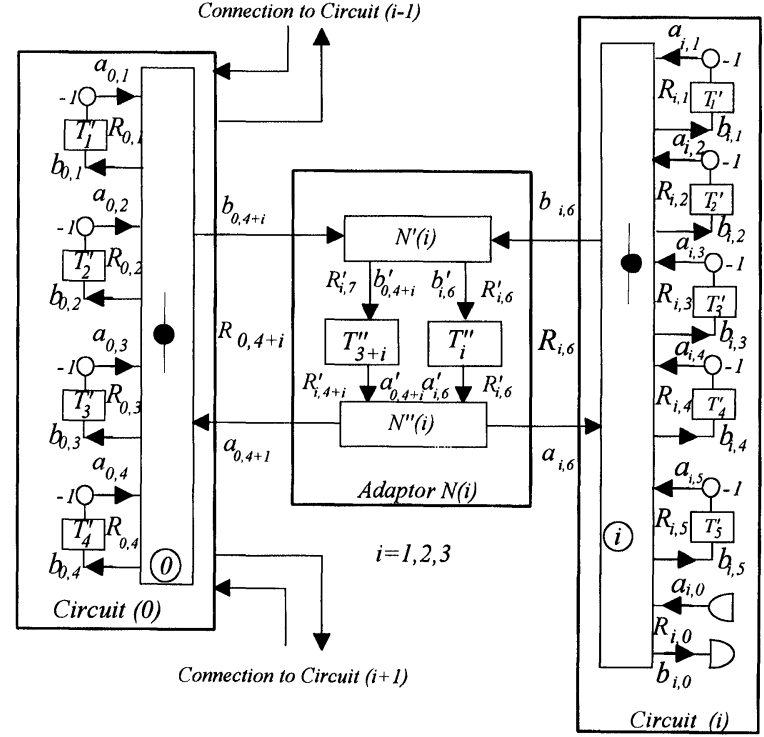

FIGURE 4 Analytic WDF arrangement.

where

$$
\begin{array}{r}
\tilde{a}_{i, 0}=\sum_{k=0}^{6} a_{i, k}, \quad \gamma_{i, k}=\frac{2 R_{i, k}}{\sum_{k=0}^{6} R_{i, k}}, \\
i=1,2,3, \quad k=0, \ldots, 6 .
\end{array}
$$

The equations of the 7-port series adaptor in the Circuit 0 are

$$
\begin{aligned}
& b_{0, k}=a_{0, k}-\gamma_{0, k} \tilde{a}_{0,0}, \quad b_{0,7}=-\left(\sum_{k=1}^{6} b_{0, k}+\tilde{a}_{0,0}\right), \\
& k=1, \ldots, 6, \\
& \left.\tilde{a}_{0,0}=\sum_{k=1}^{7} a_{0, k}, \quad \gamma_{0, k}=\frac{2 R_{0, k}}{\sum_{k=1}^{7} R_{0, k}}, \quad k=1, \ldots, 6.6 \mathrm{a}\right)
\end{aligned}
$$

We are now in position to establish the following theorem.

THEOREM 5.1 The WDF circuit realizing the Euler equations is the circuit in Figs. 3 and 4. The set of discrete MD equations numerically integrating the Euler equations is the set of Eqs. in (5.3)-(5.6).

It is convenient to express the set of MD equations numerically integrating the Euler 
equations in terms of discretized currents and voltages of the reference circuit in Fig. 2. To this end we present the general formula expressing the voltage $u$ and the current $i$ in terms of the forward and backward waves $a$ and $b$ as well as the resistance port $R$, i.e. the formula

$$
u=(a+b) \sqrt{R}, \quad i=\frac{a-b}{\sqrt{R}} .
$$

Clearly, relation (5.7) is the inverse of the relation (5.1). Expressing for each port, the port voltage in terms of the corresponding incident wave, the corresponding port resistance, and the loop current, and recalling that the sum of the loop voltages is zero, the following equations are derived

$$
\begin{aligned}
& 2 \sum_{k=0}^{5} a_{\lambda, k} \sqrt{R_{\lambda, k}}-\hat{q}_{\lambda} \sum_{k=0}^{5} R_{\lambda, k} \\
& \quad=2 a_{\lambda, 6} \sqrt{R_{\lambda, 6}}-R_{\lambda, 6} i_{\lambda, 6}, \quad \lambda=1,2,3, \\
& 2 \sum_{k=1}^{4} a_{0, k} \sqrt{R_{0, k}}-\hat{p} \sum_{k=1}^{4} R_{0, k} \\
& \quad=\sum_{k=5}^{7}\left[2 a_{0, k} \sqrt{R_{0, k}}-R_{0, k} i_{0, k}\right]
\end{aligned}
$$

where

$$
\begin{aligned}
& i_{\lambda, 6}=\left(a_{\lambda, 6}-b_{\lambda, 6}\right) / \sqrt{R_{\lambda, 6}}, \\
& i_{0, k}=\left(a_{0, k}-b_{0, k}\right) / \sqrt{R_{0, k}}, \quad \lambda=1,2,3, \quad k=5,6,7 .
\end{aligned}
$$

The set of equations in (5.8) can be expressed equivalently as follows:

$$
\begin{aligned}
2 \sum_{k=0}^{5} a_{\lambda, k} \sqrt{R_{\lambda, k}}-\hat{q}_{\lambda} \sum_{k=0}^{5} R_{\lambda, k} \\
=2 a_{0,4+\lambda}^{\prime} \sqrt{R_{0,4+\lambda}}-R_{0,4+\lambda}\left(\hat{q}_{\lambda}-\hat{p}\right) \\
\quad+2 a_{\lambda, 6}^{\prime} \sqrt{R_{\lambda, 6}}-R_{\lambda, 6}\left(\hat{q}_{\lambda}+\hat{p}\right), \quad \lambda=1,2,3 .
\end{aligned}
$$$$
2 \sum_{k=1}^{4} a_{0, k} \sqrt{R_{0, k}}-\hat{p} \sum_{k=1}^{4} R_{0, k}
$$$$
=\sum_{\lambda=1}^{3}\left[2 a_{0,4+\lambda}^{\prime} \sqrt{R_{0,4+\lambda}}-R_{0,4+\lambda}\left(\hat{q}_{\lambda}-\hat{p}\right)\right.
$$$$
\left.+2 a_{\lambda, 6}^{\prime} \sqrt{R_{\lambda, 6}}-R_{\lambda, 6}\left(\hat{q}_{\lambda}+\hat{p}\right)\right] .
$$

The set of equations in (5.9a and b) is equivalent to the set of equations in (5.5) and (5.6), respectively. The equations in (5.9) have the advantage that at any instant they have to be solved with respect to the original unknowns $\hat{p}$ and $\hat{q}_{\lambda}$ of the Euler equation (2.15).

In what follows and after substituting the port resistances by their values, the set of equations in (5.5), (5.6) and (5.9) can be rewritten as follows:

$$
\begin{aligned}
& 2 \sqrt{\frac{2}{T_{0}}}\left[\begin{array}{lllll}
a_{1,1} & a_{1,2} & a_{1,3} & a_{1,4} & a_{1,5}
\end{array}\right] \\
& \times\left[\begin{array}{c}
\left(\hat{\rho} a q_{4} \frac{q_{1}-q_{2}-q_{3}+q_{4}}{u_{0}^{2}}\right)^{1 / 2} \\
\left(\hat{\rho} a q_{4} \frac{-q_{1}+q_{2}-q_{3}+q_{4}}{u_{0}^{2}}\right)^{1 / 2} \\
\left(\hat{\rho} a q_{4} \frac{-q_{1}-q_{2}+q_{3}+q_{4}}{u_{0}^{2}}\right)^{1 / 2} \\
\left(\hat{\rho} a q_{4} \frac{q_{1}+q_{2}+q_{3}+q_{4}}{u_{0}^{2}}\right)^{1 / 2} \\
{[2(\varepsilon-1)]^{1 / 2}}
\end{array}\right] \\
& -\frac{4 q_{1}}{q_{4} T_{0}^{\prime} \sqrt{a}}\left[\frac{4 \hat{\rho} a q_{4}^{2}}{u_{0}^{2}}-2(\varepsilon+1)\right] \\
& +2 a_{1,0}\left(\frac{\hat{\rho} a^{4} q_{4}^{2}}{u_{0}^{2} T_{0}^{\prime}}-2 \varepsilon\right)^{1 / 2} \\
& -\frac{8}{T_{0}^{\prime}}\left[a_{0,5}^{\prime} \frac{\sqrt{T_{0}^{\prime}}}{2}+a_{1,6}^{\prime} \frac{\sqrt{T_{0}^{\prime}}}{2}-\frac{q_{1}}{q_{4} \sqrt{a}}\right]=0 .
\end{aligned}
$$

$$
\begin{aligned}
& 2 \sqrt{\frac{2}{T_{0}}}\left[\begin{array}{lllll}
a_{2,1} & a_{2,2} & a_{2,3} & a_{2,4} & a_{2,5}
\end{array}\right] \\
& \times\left[\begin{array}{c}
\left(\hat{\rho} a q_{4} \frac{q_{1}-q_{2}-q_{3}+q_{4}}{u_{0}^{2}}\right)^{1 / 2} \\
\left(\hat{\rho} a q_{4} \frac{-q_{1}+q_{2}-q_{3}+q_{4}}{u_{0}^{2}}\right)^{1 / 2} \\
\left(\hat{\rho} a q_{4} \frac{-q_{1}-q_{2}+q_{3}+q_{4}}{u_{0}^{2}}\right)^{1 / 2} \\
\left(\hat{\rho} a q_{4} \frac{q_{1}+q_{2}+q_{3}+q_{4}}{u_{0}^{2}}\right)^{1 / 2} \\
{[2(\varepsilon-1)]^{1 / 2}}
\end{array}\right]
\end{aligned}
$$




$$
\begin{aligned}
& -\frac{4 q_{2}}{q_{4} T_{0}^{\prime} \sqrt{a}}\left[\frac{4 \hat{\rho} a q_{4}^{2}}{u_{0}^{2}}-2(\varepsilon+1)\right] \\
& +2 a_{2,0}\left(\frac{\hat{\rho} a^{4} q_{4}^{2}}{u_{0}^{2} T_{0}^{\prime}}-2 \varepsilon\right)^{1 / 2} \\
& -\frac{8}{T_{0}^{\prime}}\left[a_{0,6}^{\prime} \frac{\sqrt{T_{0}^{\prime}}}{2}+a_{2,6}^{\prime} \frac{\sqrt{T_{0}^{\prime}}}{2}-\frac{q_{2}}{q_{4} \sqrt{a}}\right]=0 \text {. } \\
& 2 \sqrt{\frac{2}{T_{0}}}\left[\begin{array}{lllll}
a_{3,1} & a_{3,2} & a_{3,3} & a_{3,4} & a_{3,5}
\end{array}\right] \\
& \times\left[\begin{array}{c}
\left(\hat{\rho} a q_{4} \frac{q_{1}-q_{2}-q_{3}+q_{4}}{u_{0}^{2}}\right)^{1 / 2} \\
\left(\hat{\rho} a q_{4} \frac{-q_{1}+q_{2}-q_{3}+q_{4}}{u_{0}^{2}}\right)^{1 / 2} \\
\left(\hat{\rho} a q_{4} \frac{-q_{1}-q_{2}+q_{3}+q_{4}}{u_{0}^{2}}\right)^{1 / 2} \\
\left(\hat{\rho} a q_{4} \frac{q_{1}+q_{2}+q_{3}+q_{4}}{u_{0}^{2}}\right)^{1 / 2} \\
{[2(\varepsilon-1)]^{1 / 2}}
\end{array}\right] \\
& -\frac{4 q_{3}}{q_{4} T_{0}^{\prime} \sqrt{a}}\left[\frac{4 \hat{\rho} a q_{4}^{2}}{u_{0}^{2}}-2(\varepsilon+1)\right] \\
& +2 a_{3,0}\left(\frac{\hat{\rho} a^{4} q_{4}^{2}}{u_{0}^{2} T_{0}^{\prime}}-2 \varepsilon\right)^{1 / 2} \\
& -\frac{8}{T_{0}^{\prime}}\left[a_{0,7}^{\prime} \frac{\sqrt{T_{0}^{\prime}}}{2}+a_{3,6}^{\prime} \frac{\sqrt{T_{0}^{\prime}}}{2}-\frac{q_{3}}{q_{4} \sqrt{a}}\right]=0 \text {. }
\end{aligned}
$$

$$
\begin{aligned}
& 2 \sqrt{\frac{2}{T_{0}^{\prime}}}\left[\begin{array}{llll}
a_{0,1} & a_{0,2} & a_{0,3} & a_{0,4}
\end{array}\right] \\
& \times\left[\begin{array}{c}
\left(2 \frac{\nu \hat{\rho}-1}{\hat{p}} \frac{q_{1}-q_{2}-q_{3}+q_{4}}{\sqrt{a} q_{4}}-3+\frac{K_{1}}{\hat{p}^{2}}-\varepsilon\right)^{1 / 2} \\
\left(2 \frac{\nu \hat{\rho}-1}{\hat{p}} \frac{-q_{1}+q_{2}-q_{3}+q_{4}}{\sqrt{a} q_{4}}-3+\frac{K_{1}}{\hat{p}^{2}}-\varepsilon\right)^{1 / 2} \\
\left(2 \frac{\nu \hat{\rho}-1}{\hat{p}} \frac{-q_{1}-q_{2}+q_{3}+q_{4}}{\sqrt{a} q_{4}}-3+\frac{K_{1}}{\hat{p}^{2}}-\varepsilon\right)^{1 / 2} \\
\left(2 \frac{\nu \hat{\rho}-1}{\hat{p}} \frac{q_{1}+q_{2}+q_{3}+q_{4}}{\sqrt{a} q_{4}}-3+\frac{K_{1}}{\hat{p}^{2}}-\varepsilon\right)^{1 / 2}
\end{array}\right]
\end{aligned}
$$

$$
\begin{aligned}
& a_{i, 1}\left(t^{\prime}\right)=b_{i, 1}\left(t^{\prime}-T_{1}^{\prime}\right), i=0,1,2,3, \\
& a_{i, 2}\left(t^{\prime}\right)=b_{i, 2}\left(t^{\prime}-T_{2}^{\prime}\right), \quad i=0,1,2,3, \\
& a_{i, 3}\left(t^{\prime}\right)=b_{i, 3}\left(t^{\prime}-T_{3}^{\prime}\right), \quad i=0,1,2,3, \\
& a_{i, 4}\left(t^{\prime}\right)=b_{i, 4}\left(t^{\prime}-T_{4}^{\prime}\right), \quad i=0,1,2,3, \\
& a_{i, 5}\left(t^{\prime}\right)=b_{i, 5}\left(t^{\prime}-T_{5}^{\prime}\right), \quad i=1,2,3 . \\
& a_{0,4+i}\left(t^{\prime}\right)=-\frac{1}{2}\left(b_{0,4+i}+b_{i, 6}\right)\left(t^{\prime}-T_{3+i}^{\prime \prime}\right) \\
& \quad+\frac{1}{2}\left(b_{0,4+i}-b_{i, 6}\right)\left(t^{\prime}-T_{i}^{\prime \prime}\right), \\
&-\frac{1}{2}\left(b_{0,4+i}+b_{i, 6}\right)\left(t^{\prime}-T_{3+i}^{\prime \prime}\right) \\
& \quad-\frac{1}{2}\left(b_{0,4+i}-b_{i, 6}\right)\left(t^{\prime}-T_{i}^{\prime \prime}\right) .
\end{aligned}
$$

Clearly, all variables in (5.10a-d) are functions of the generalized time $t^{\prime}$ except the constants $T_{0}^{\prime}, a$ and $\varepsilon$, which has to be specified for the faster convergence of the algorithm.

Based upon the above system of equations the following theorem is established.

THEOREM 5.2 The MD WDF integration of the Euler equations is the set of nonlinear $M D$ equations in (5.10) and (5.7).

From the computational point of view, the problem of solving the Euler equations has been reduced to that of solving a nonlinear algebraic system of equations.

\section{CONCLUSIONS}

In this paper the problem of numerically integrating the Euler equations has been studied. A numerically stable and numerically robust discretization has been derived, via appropriate passive 
WDF realizations. The analytic expression of the nonlinear discrete equations, approximating the Euler equations, is determined. The nonlinear MD WDF equations numerically integrating the Euler equations are analytically determined. The present results appear to contribute to the numerical solution of many fluid dynamic problems as well as in controlling distributed parameter flight systems. Work is under progress for the expression of physical properties of the Euler equations in terms of the respective MD WDF equations.

\section{Acknowledgment}

The authors would like to thank Prof. A. Fettweis for his encouragement, useful comments and fruitful discussions.

\section{References}

[1] R.D. Richtmyer and K.W. Morton, Difference method for initial-value problems, Interscience, New York, 1967.

[2] R.C. LeBail, "Use of fast Fourier transform for solving partial differential equations", J. Comput. Phys., 9, $440-465,1972$.

[3] K. Gustafson and K. Halasi, "Cavity flow dynamics at higher Reynolds number and higher aspect ratios", J. Comput. Phys., 70, 271-283, 1979.
[4] S.F. McCormick, Multilevel Adaptive Methods for Partial Differential Equations, SIAM Studies in Applied Mathematics, 1989.

[5] S.F. McCormick, Multilevel Projection Methods for Partial Differential Equations, SIAM Studies in Applied Mathematics, 1992.

[6] R.E. Bank, PLTMG: A Software Package for Solving Elliptic Partial Differential Equations, SIAM Studies in Applied Mathematics, 1994.

[7] R. Peyret and D.T. Taylor, Computational Methods for Fluid Flow, Springer Verlag, New York, 1983.

[8] M. Prestin and L. Shtilman, "A parallel Navier-Stokes solver: The Meikp implementation", J. Supercomputing, 9, 347-364, 1997.

[9] A. Fettweis, "Discrete modeling of losseless fluid dynamic systems", $A E \ddot{U}$, 46, 209-218, 1992.

[10] R. Bernhardt and D. Dahlhaus, "Numerical integration of the Euler equations by means of wave digital filters", ICASSP, Australia, 1994.

[11] A. Fettweis, "Wave digital filters: Theory and practice", Proc. IEEE, 74, 270-327, 1986.

[12] A. Fettweis, "On assessing robustness of recursive digital filters", European Transactions on Telecommunications, 1, 103-109, 1990.

[13] A. Fettweis, "Multidimensional wave digital filters for discrete-time modeling of Maxwell's equations", Int. J. Num. Modeling, 5, 1-29, 1992.

[14] A. Fettweis and G. Nitsche, "Numerical integration of partial differential equations using principles of multidimensional wave digital filters", Journal of VLSI Signal Proc., 3, 7-24, 1991.

[15] A. Fettweis and G. Nitsche, "Numerical integration of partial differential equations by means of multidimensional wave digital filters", Proc. IEEE Int. Symp. Circuits and Systems, Vol. 2, New Orleans, LA., May 1990, pp. 954-957.

[16] F.M. Whitaker, Introduction to Fluid Mechanics, PrenticeHall, Engelwood Cliffs, N.J., 1968. 


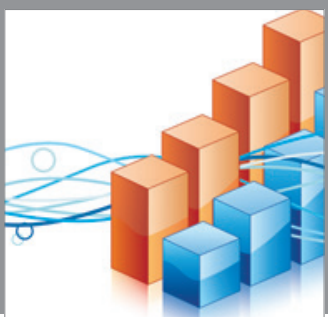

Advances in

Operations Research

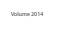

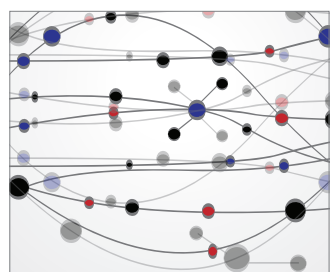

\section{The Scientific} World Journal
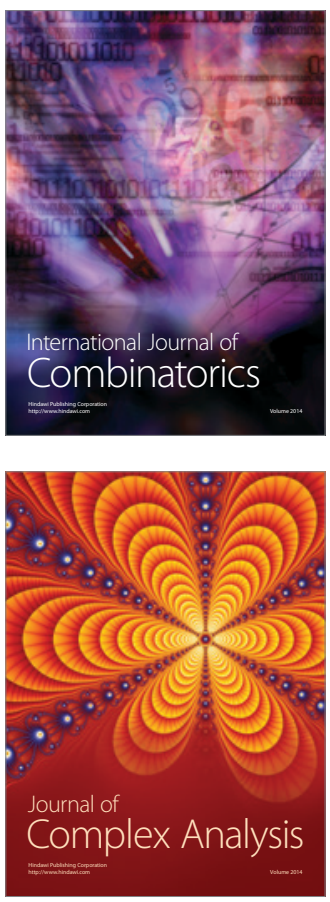

International Journal of

Mathematics and

Mathematical

Sciences
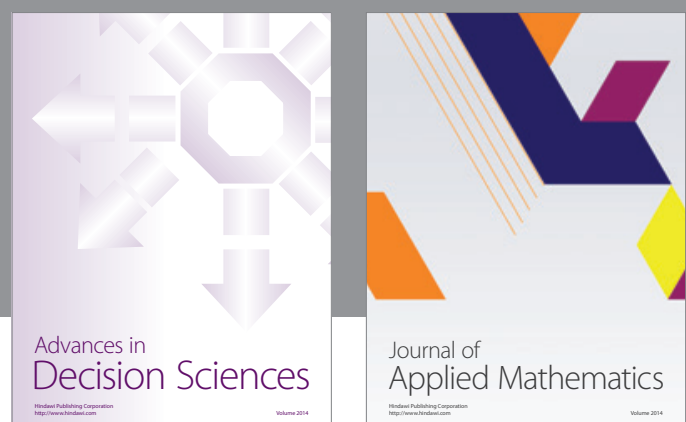

Journal of

Applied Mathematics
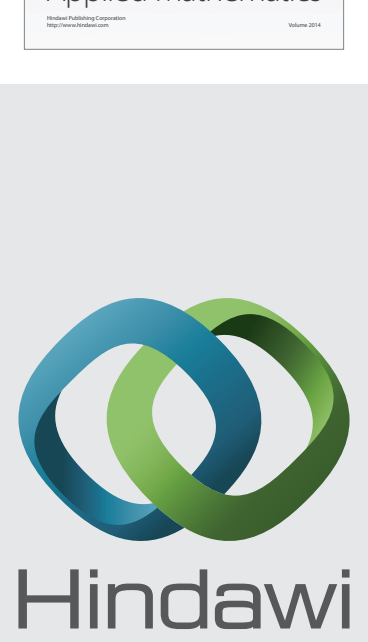

Submit your manuscripts at http://www.hindawi.com
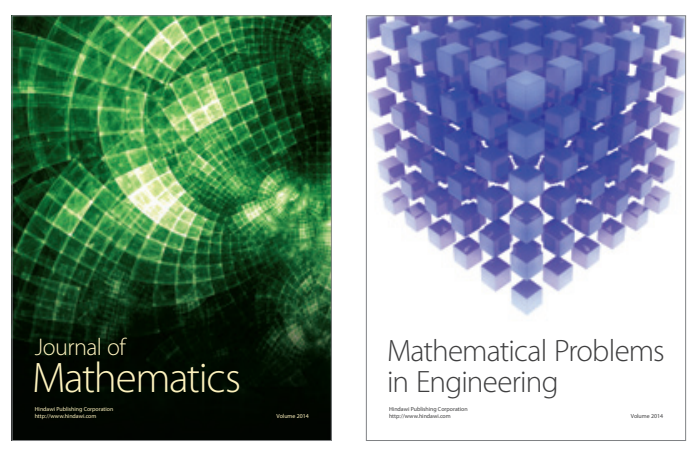

Mathematical Problems in Engineering
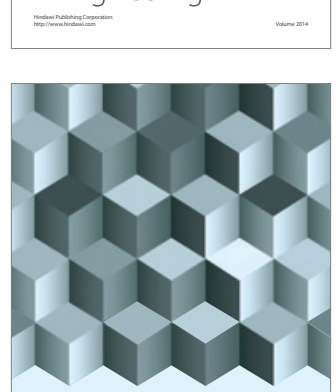

Journal of

Function Spaces
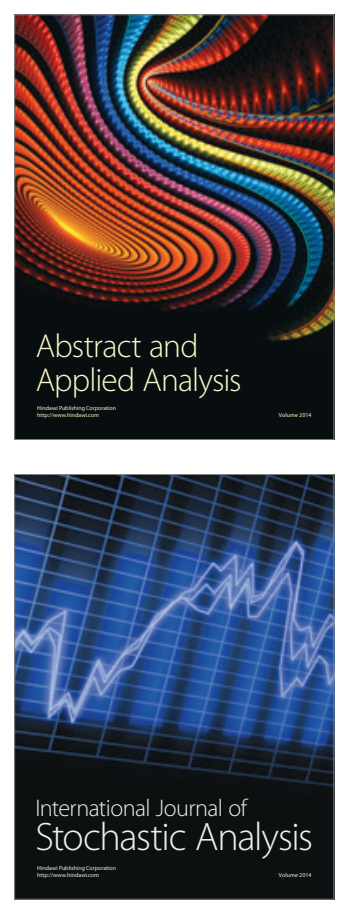

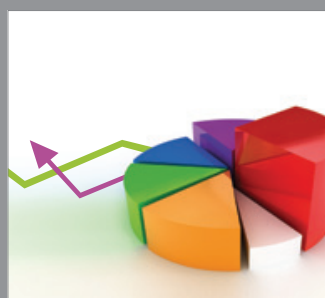

ournal of

Probability and Statistics

Promensencen
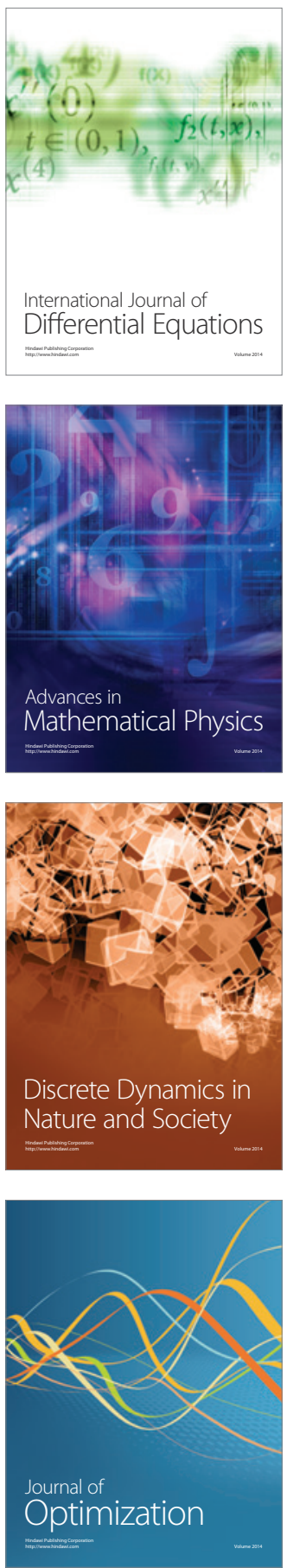